\title{
ASSOCIATION BETWEEN PARKINSON'S DISEASE AND DENTAL AMALGAM FILLING: A STUDY OF NATIONWIDE POPULATION-BASED CASE CONTROL IN TAIWAN
}

\author{
CHEN, K. H. \\ Department of Artificial Intelligence, CTBC Business School \\ No. 600, Sec. 3, Taijiang Blvd., Annan District, Tainan 709, Taiwan, R.O.C. \\ e-mail: khchen@ctbc.edu.tw; phone: +886-6-287-3335; fax: +886-6-287-3536 \\ (Received 10 $0^{\text {th }}$ Apr 2020; accepted $29^{\text {th }}$ Jul 2020)
}

\begin{abstract}
Parkinson's disease (PD) is a common associated motor dysfunction nervous system disease. Low-level occupational mercury vapor exposure is a commonly known critical factor that increases the risk of PD. Dental amalgams, used for dental restoration, are a major source of mercury exposure for people. However, the relationship between PD and the use of amalgam fillings (AMFs) has yet to be clarified. This paper of retrospective case-control aimed to examine the relationship between AMF use and the risk of PD; it analyzed Taiwan's NHIRD (National Health Insurance Research Database) based on population administrative database, for the 2000-2013 period. For the case and control groups, Charlson Comorbidity Index, age, urbanization level, sex and monthly income were all matched by a propensity score method for a 1:1 ratio; 5712 cases and 5712 controls participated in this study. This results of case-control based on nationwide population revealed no significant association between PD and AMF in Taiwanese people. This result provides meaningful implications despite being statistically not significant.
\end{abstract}

Keywords: dental amalgam, mercury, NHIRD, nationwide population, case-control study

\section{Introduction}

Parkinson's disease (PD) is a neurodegenerative disease. The incidence rate among women aged younger than 60 years is 8.6 per 100,000 people; this figure increases to 29 and 78.4 per 100,000 people among women aged 60-69 and 70-79 years old, respectively. The incidence rate among men aged younger than 60 years is 11.1 per 100,000 people; this figure increases to 49.5 and 140.7 among those aged 60-69 and 70-79 years old, respectively (Van Den Eeden et al., 2003). The risk and prevalence rate of PD increases with age (Bower et al., 1999; Baldereschi et al., 2000). With respect to prevalence rate, $\mathrm{PD}$, as a progressive neurodegenerative disease, is second only to Alzheimer's disease (Wirdefeldt et al., 2011). The main clinical symptoms of PD are mostly associated with motor dysfunction-including rigidity, resting tremor, bradykinesia, and postural instability (Frank et al., 2006; Davie, 2008). Generic and environmental risk factors may cause the dopaminergic neurons death in the brain, where these dead neurons, in turn, inhibit the neural pathways from the basal ganglia to the motor cortex (Davie, 2008).

Dental amalgams are a common dental filling material with a $50 \%$ mercury content. Traditional low-copper amalgams have a copper content lower than 6\%. The high-copper dental amalgams in the 1960s were developed with a copper content of 8\%-10\% (Haque et al., 2019). In high-copper dental amalgams, mercury is more soluble under acidic conditions relative to its low-copper counterpart (Okabe et al., 2003). High-copper amalgams, because of the amount of mercury vapor released from it (Bengtsson and Hylander, 2017), has a higher corrosion rate and longer corrosion time relative to early amalgams that contain metal composites. 
Autopsy reports have demonstrated that dental amalgam fillings (AMFs) cause mercury accumulation in the human body (Mutter, 2011). AMF use has been found to correlate with mercury content in the brain and kidney tissues (Eggleston and Nylander, 1987; Nylander et al., 1987; Barregård et al., 1999; Guzzi et al., 2006) and to positively correlate with mercury content in the blood and urine (Kingman et al., 1998; Zimmer et al., 2002; Levy et al., 2004). A recent epidemiological study with 2137 participants revealed that relative to those who had not, people who had had 7 or more of their teeth filled with AMF had a 30\%-50\% higher mercury content in their urine (Dutton et al., 2013).

The pathogenesis of PD has yet to be elucidated. Several case reports and epidemiological studies have indicated that exposure to certain metals long-term (e.g., aluminum, manganese, copper, iron, mercury, lead, zin, and those in amalgams) is a potential factor of risk for PD. The PD development may be associated with the dose of mercury exposure (Ngim and Devathasan, 1989). However, studies have also determined that working in jobs involving long-term exposure to manganese, mercury, or aluminum has no effect on PD development (Semchuk et al., 1993; Vieregge et al., 1995). Accordingly, to control the incidence rate of PD, the study used the Database of National Health Insurance Research Database (NHIRD) to investigate the relationship between PD development and amalgam use.

\section{Materials and Methods}

\section{Study Design and Data Source}

The data used in this paper were collected from the 2010 Longitudinal Health Insurance Database (2010LHID), a publicly accessible database compiled by Taiwan's National Health Research Institutes. LHID2010 contains raw insurance claims data and registration documents for 2000-2013, including the information of 1 million people selected randomly from beneficiaries registered compulsory National Health Insurance (NHI) procedure in Taiwan, which covered $99.9 \%$ of the Taiwanese population in 2010 (Charlson et al., 1987). The present study defined diagnoses corresponding to the ICD-9-CM (International Classification of Diseases, Ninth Revision, Clinical Modification). Because data from the LHID 2010 were used for research and were deidentified secondary data, therefore the consent of written informed was not required.

\section{Control Groups and Selection of Case}

This study limiting is the adult population, the present case-control study excluded those aged younger than 20 years, those who had withdrawn from the NHI procedure, or those with missing data in the 2010LHID. Additionally, only individuals who had received three or more consensus diagnoses of PD were included in the sample to improve the diagnostic effectiveness for PD in the LHID2010. Propensity score matching by a 1:1 ratio was performed to conditionally select participants in the population for comparison according to urbanization level, the Charlson Comorbidity Index (CCI) (Charlson et al., 1994), age, socioeconomic status, and sex. The CCI ranges from 0 to 3 , with a high CCI indicating a high risk of mortality for a particular patient (diabetes, hypertension, etc.) (Charlson et al., 1987). Figure 1 illustrates the research process. 


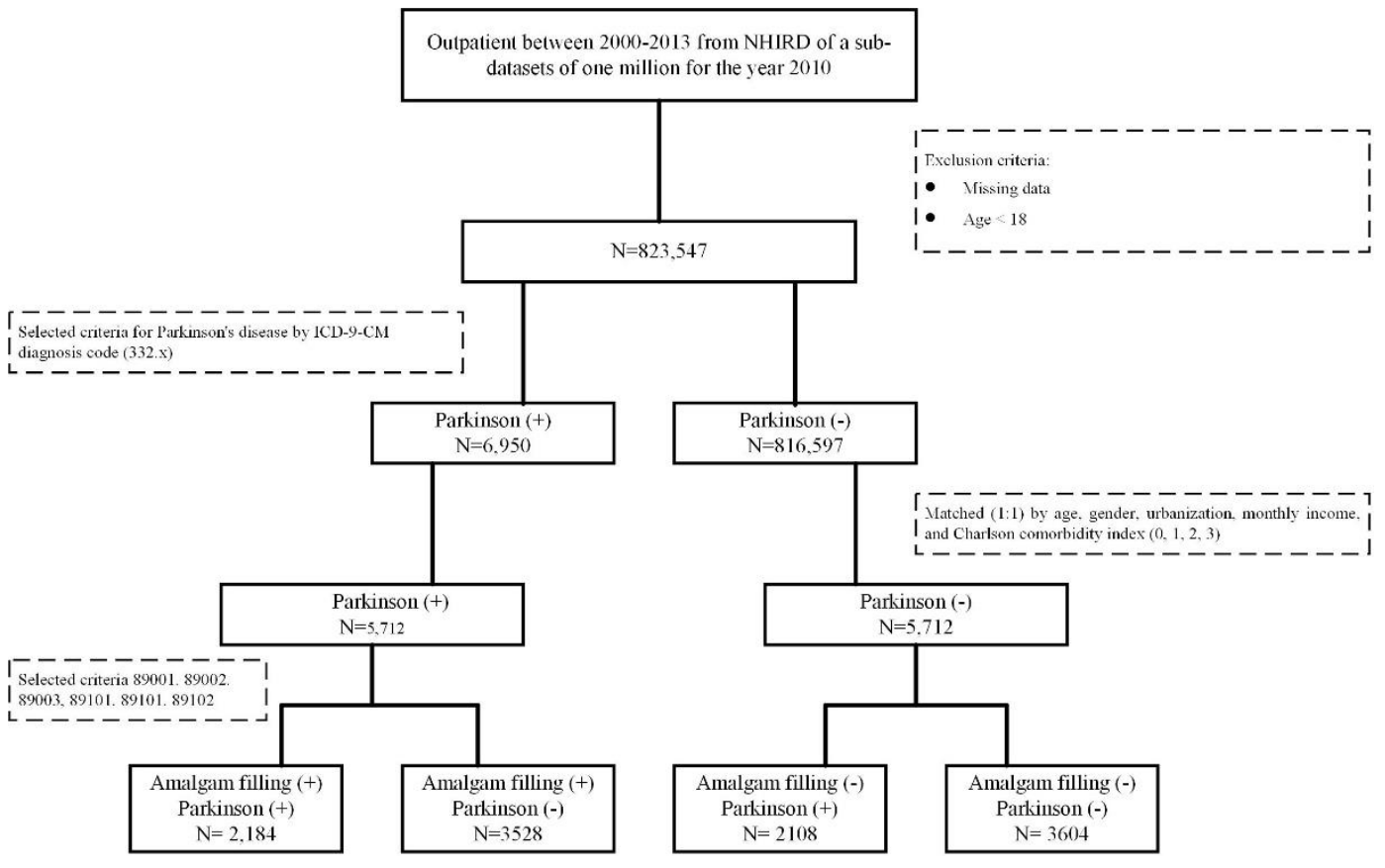

Figure 1. The flowchart of the study

\section{Exposure Assessment}

This study identified patients, from the LHID2010, who had received AMF-related treatments according to the following procedure codes: Dental amalgam fillings (89001C, 89002C, 89003C) and other dental treatments (89101C, 89102C, 89103C) (Table 1). Confounding factors - namely age, urbanization level, CCI, socioeconomic factors, and sex-were included in the analysis (Figure 1).

Table 1. The amalgam filling codes

\begin{tabular}{|c|c|c|c|}
\hline \multicolumn{2}{|r|}{ Dental amalgam fillings } & \multicolumn{2}{|r|}{ Other dental treatments } \\
\hline Code & Treatment & Code & Treatment \\
\hline $89001 \mathrm{C}$ & Amalgam restoration for single-face. & $89101 \mathrm{C}$ & $\begin{array}{l}\text { Amalgam restoration for single-surface in } \\
\text { specific cases. }\end{array}$ \\
\hline $89002 \mathrm{C}$ & Amalgam restoration for two-face. & $89102 \mathrm{C}$ & $\begin{array}{l}\text { Amalgam restoration for two- surface in } \\
\text { specific cases. }\end{array}$ \\
\hline $89003 \mathrm{C}$ & Amalgam restoration for three-face. & $89103 \mathrm{C}$ & $\begin{array}{l}\text { Amalgam restoration three-surface in } \\
\text { specific cases. }\end{array}$ \\
\hline
\end{tabular}

\section{Statistical Analysis}

SPSS 18 (International Business Machines Corporation, Chicago, IL, United States) was used for data analyses. For the continuous variables, this study used Student's $t$-test; for categorical variables, this study used a chi-square test, and the odds ratio (OR) for calculating between the control and case groups; and multiple logistic regression was used in a stratified analysis. All results are presented in terms of the OR and $95 \%$ confidence interval $(95 \% \mathrm{CI})$. Sex, region of residence, age, CCI, and income were adjusted for in the analyses. Statistical significance was defined when the p-value was $<0.05$. 


\section{Result}

This study recruited 5712 cases and 5712 controls. As listed in Table 2, the average age was $76.87 \pm 11.73$ years old; $5806(50.82 \%)$ and $5618(49.18 \%)$ of them were women and men, respectively. The case and control groups did not significantly differ with respect to their demographic characteristics, specifically their sex, region of residence, CCI, age, urbanization level, and monthly income $(P>0.05)$.

Table 2. The individual's demographic characteristics in this research

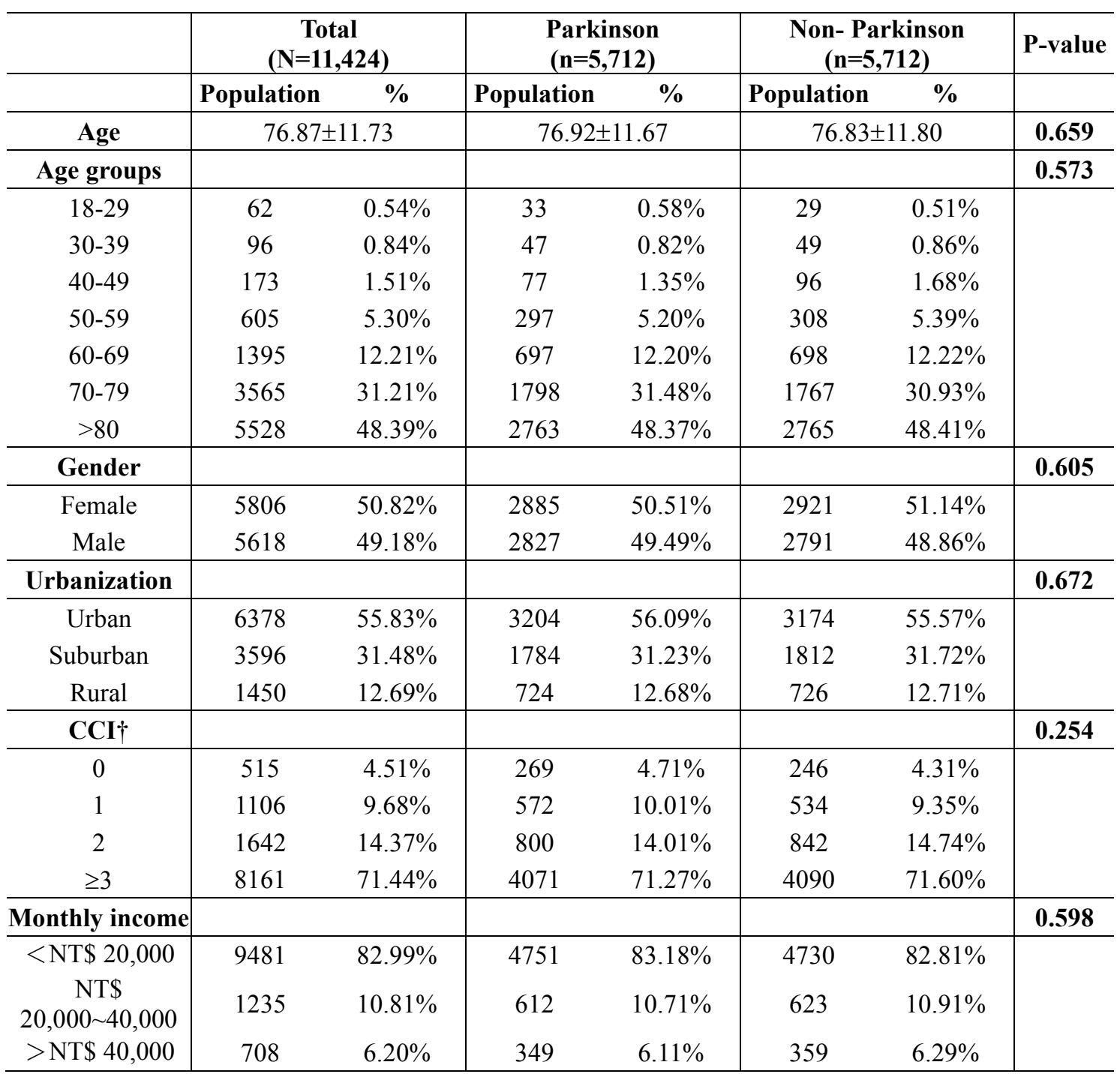

$\dagger$ Charlson comorbidity index

As presented in Table 3, PD and AMF were not directly related (adjusted OR $=1.067$, THE CI of 95\%: 0.99-1.15).

Table 4 illustrates the gender -stratified OR for PD and AMF. With respect to sex, no PD and AMF were not associated. The adjusted OR for AMF and PD for men and women were 1.096 (The CI of 95\%: 0.984-1.211) and 1.023 (The CI of 95\%: 0.920-1.138), respectively. 
Table 3. The odds ratio between the amalgam filling and Parkinson's disease

\begin{tabular}{c|cc|cc}
\hline & \multicolumn{2}{|c|}{ With Parkinson $(\mathbf{n = 5 , 7 1 2})$} & \multicolumn{2}{c}{ Without Parkinson (n=5,712) } \\
\hline & Number of patients & \% & Number of patients & \% \\
\hline AMF & 2184 & $38.24 \%$ & 2108 & $36.90 \%$ \\
Non-AMF & 3528 & $61.76 \%$ & 3604 & $63.10 \%$ \\
\hline OR (95\% CI) & \multicolumn{2}{|c|}{$1.058(0.981-1.142)$} & 1.00 & \\
Adjusted OR (95\% CI) & \multicolumn{2}{|c|}{$1.067(0.987-1.153)$} & \multicolumn{2}{c}{1.00} \\
\hline
\end{tabular}

Abbreviations: PD—Parkinson's disease; OR—odds ratio; AMF—amalgam filling; $\mathrm{CI}$-confidence interval. Adjustment by gender, CCI, urbanization, monthly income, and age; $* p<0.05$

Table 4. Odds ratio for gender of those with a diagnosis of Parkinson's disease with an amalgam filling

\begin{tabular}{|c|c|c|c|c|}
\hline Sex & $\begin{array}{c}\text { With PD } \\
\text { Number of AMF }\end{array}$ & $\begin{array}{c}\text { Without PD } \\
\text { Number of AMF }\end{array}$ & $\begin{array}{c}\text { OR } \\
(95 \% \mathrm{CI})\end{array}$ & $\begin{array}{c}\text { Adjusted OR } \\
\text { (95\% CI) }\end{array}$ \\
\hline Female & 1092 & 1793 & $\begin{array}{c}1.023 \\
(0.920-1.138)\end{array}$ & $\begin{array}{c}1.043 \\
(0.936-1.163)\end{array}$ \\
\hline Male & 1092 & 1735 & $\begin{array}{c}1.096 \\
(0.984-1.211)\end{array}$ & $\begin{array}{c}1.098 \\
(0.984-1.225)\end{array}$ \\
\hline
\end{tabular}

Abbreviations: PD—Parkinson's disease; OR—odds ratio; AMF—amalgam filling; $\mathrm{CI}$-confidence interval. Adjustment by urbanization, CCI, age, and monthly income; * $p<0.05$

\section{Discussion}

As far as authors' know, the present study is the first to examine the association between PD and AMF based on a large-scale population-based database and tracking time 13 years. The results revealed no positive correlation between AMF and PD. To ensure accurate PD diagnosis, this study recruited only patients with at least three PD diagnoses. The effectiveness of the PD diagnoses was validated through the NHI system of conducting regular verification of diagnoses; such verification is part of the NHI's treatment guidelines to ensure that health insurance claims are legitimate. The findings of this registry-based study are significant and meaningful.

Dental amalgams of all types contain mercury, which is released in the form of mercury vapor. Mercury's toxicity can cause cell damage through the increased production of free radicals (Olivieri et al., 2000). Heavy metals are suspected to cause the generation of free radicals, which increases oxidative stress in neurons, which, in turn, causes PD. Usually, nervous system diseases are the body accumulation of heavy metals or associated with environmental exposure. Heavy metals can affect the basal ganglia in the brain, which causes physical derangement through damaging neurons in the substantia nigra pars compacta. PD is a highly common motor disorder (Montgomery, 1995). Case reports and epidemiological studies have reported long-term exposure to mercury to be a potential risk factor for PD (Ngim and Devathasan, 1989), whereas some studies have revealed no association between the two (Semchuk et al., 1993; Vieregge et al., 1995). In the present study, PD and AMF use were not positively correlated. This is attributable to possibly differing levels of mercury exposure in occupational exposure than in exposure from AMF use. Mercury can leach out from the surface of AMFs when chewing and tooth brushing. However, this study did not assess the extent of exposure to mercury vapor in patients using AMFs. 
This study has some limitations. First, the NHIRD provides no data on family medical history, symptoms, PD severity, dietary habits, and the brand and formula of amalgam used; such missing information makes the analysis robust, especially with regard to controlling for confounders. Second, the procedure codes in the NHI did not indicate the positions of AMFs. Third, this study did not collect information on the use of other metal dental restoration implements (e.g., dental inlays or crowns) from the NHIRD. Fourth, propensity score matching cannot cope with unobservable confounders, which limits the analysis of this study.

\section{Conclusions}

On the basis of results for a nationwide population, the present study revealed no association between PD and AMF in Taiwanese people. Dental amalgams are a potential source of environmental mercury exposure, and it must be investigated as a possible contributor to mercury-induced neurotoxicity. An in-depth risk-benefit analysis on the use of AMF must include the effects, service life, and performance of dental amalgams. Overall, despite dental amalgam fillings use very widespread, the safety data of dental amalgam fillings safety are inadequate. Most reassurance is provided for Parkinson's disease in this study. The further, most in need investigation are effects and neurodegenerative diseases on children and infants.

\section{REFERENCES}

[1] Baldereschi, M., Di Carlo, A., Rocca, W. A., Vanni, P., Maggi, S., Perissinotto, E., Grigoletto, F., Amaducci, L., Inzitari, D. (2000): Parkinson's disease and parkinsonism in a longitudinal study: two-fold higher incidence in men. - Neurology 55(9): 1358-1363.

[2] Barregård, L., Svalander, C., Schütz, A., Westberg, G., Sällsten, G., Blohmé, I., Mölne, J., Attman, P. O., Haglind, P. (1999): Cadmium, mercury, and lead in kidney cortex of the general Swedish population: a study of biopsies from living kidney donors. Environmental Health Perspectives 107(11): 867-871.

[3] Bengtsson, U. G., Hylander, L. D. (2017): Increased mercury emissions from modern dental amalgams. - Biometals 30(2): 277-283.

[4] Bower, J. H., Maraganore, D. M., McDonnell, S. K., Rocca, W. A. (1999): Incidence and distribution of parkinsonism in Olmsted County, Minnesota, 1976-1990. - Neurology 52(6): 1214-1214.

[5] Charlson, M. E., Pompei, P., Ales, K. L., MacKenzie, C. R. (1987): A new method of classifying prognostic comorbidity in longitudinal studies: development and validation. Journal of chronic diseases 40(5): 373-383.

[6] Charlson, M., Szatrowski, T. P., Peterson, J., Gold, J. (1994): Validation of a combined comorbidity index. - Journal of clinical epidemiology 47(11): 1245-1251.

[7] Davie, C. A. (2008): A review of Parkinson's disease. - British medical bulletin 86(1): 109127.

[8] Dutton, D. J., Fyie, K., Faris, P., Brunel, L., Emery, J. H. (2013): The association between amalgam dental surfaces and urinary mercury levels in a sample of Albertans, a prevalence study. - Journal of occupational medicine and toxicology 8(1): 22.

[9] Eggleston, D. W., Nylander, M. (1987): Correlation of dental amalgam with mercury in brain tissue. - Journal of Prosthetic Dentistry 58(6): 704-707.

[10] Frank, C., Pari, G., Rossiter, J. P. (2006): Approach to diagnosis of Parkinson disease. Canadian Family Physician 52(7): 862-868. 
[11] Guzzi, G., Grandi, M., Cattaneo, C., Calza, S., Minoia, C., Ronchi, A., Gatti, A., Severi, G. (2006): Dental amalgam and mercury levels in autopsy tissues: food for thought. - The American journal of forensic medicine and pathology 27(1): 42-45.

[12] Haque, N., Yousaf, S., Nejatian, T., Youseffi, M., Mozafari, M., Sefat, F. (2019): Dental amalgam. - In: Khurshid, Z., Najeeb, S., Zafar, M. S., Sefat, F. (eds.) Advanced Dental Biomaterials. Woodhead Publishing, pp. 105-125.

[13] Kingman, A., Albertini, T., Brown, L. J. (1998): Mercury concentrations in urine and whole blood associated with amalgam exposure in a US military population. - Journal of Dental Research 77(3): 461-471.

[14] Levy, M., Schwartz, S., Dijak, M., Weber, J. P., Tardif, R., Rouah, F. (2004): Childhood urine mercury excretion: dental amalgam and fish consumption as exposure factors. Environmental Research 94(3): 283-290.

[15] Montgomery Jr, E. B. (1995): Heavy metals and the etiology of Parkinson's disease and other movement disorders. - Toxicology 97(1-3): 3-9.

[16] Mutter, J. (2011): Is dental amalgam safe for humans? The opinion of the scientific committee of the European Commission. - Journal of Occupational Medicine and Toxicology 6(1): 2 .

[17] National Health Insurance Administration. (2015): National Health Insurance Annual Report 2015-2016. - Ministry of Health and Welfare.

[18] Ngim, C. H., Devathasan, G. (1989): Epidemiologic study on the association between body burden mercury level and idiopathic Parkinson's disease. - Neuroepidemiology 8(3): 128141.

[19] Nylander, M., Friberg, L., Lind, B. (1987): Mercury concentrations in the human brain and kidneys in relation to exposure from dental amalgam fillings. - Swedish dental journal 11(5): 179-187.

[20] Okabe, T., Elvebak, B., Carrasco, L., Ferracane, J. L., Keanini, R. G., Nakajima, H. (2003): Mercury release from dental amalgams into continuously replenished liquids. - Dental Materials 19(1): 38-45.

[21] Olivieri, G., Brack, C., Müller-Spahn, F., Stähelin, H. B., Herrmann, M., Renard, P., Brockhaus, M., Hock, C. (2000): Mercury induces cell cytotoxicity and oxidative stress and increases $\beta$-amyloid secretion and tau phosphorylation in SHSY5Y neuroblastoma cells. - Journal of Neurochemistry 74(1): 231-236.

[22] Semchuk, K. M., Love, E. J., Lee, R. G. (1993): Parkinson's disease: a test of the multifactorial etiologic hypothesis. - Neurology 43(6): 1173-1173.

[23] Van Den Eeden, S. K., Tanner, C. M., Bernstein, A. L., Fross, R. D., Leimpeter, A., Bloch, D. A., Nelson, L. M. (2003): Incidence of Parkinson's disease: variation by age, gender, and race/ethnicity. - American journal of epidemiology 157(11): 1015-1022.

[24] Vieregge, P., Heinzow, B., Korf, G., Teichert, H. M., Schleifenbaum, P., Mösinger, H. U. (1995): Long term exposure to manganese in rural well water has no neurological effects. - Canadian journal of neurological sciences 22(4): 286-289.

[25] Wirdefeldt, K., Adami, H. O., Cole, P., Trichopoulos, D., Mandel, J. (2011): Epidemiology and etiology of Parkinson's disease: a review of the evidence. - European journal of epidemiology 26(1): 1 .

[26] Zimmer, H., Ludwig, H., Bader, M., Bailer, J., Eickholz, P., Staehle, H. J., Triebig, G. (2002): Determination of mercury in blood, urine and saliva for the biological monitoring of an exposure from amalgam fillings in a group with self-reported adverse health effects. - International journal of hygiene and environmental health 205(3): 205-211. 